\title{
Swappable Distributed MIMO Controller for a VCT Engine
}

\author{
Melih Çakmakcı and A. Galip Ulsoy
}

\begin{abstract}
In the early days of computer control, only one centralized computer was responsible for executing the algorithms. Increasingly, computer control algorithms reside inside individual system components in a distributed fashion. Variable camshaft timing (VCT) is an appealing feature for automotive engines because it allows optimization of the cam timing over a wide range of operating conditions. In this paper, a method to distribute the discrete multiple-input mutiple-output controller for the VCT engine to improve the component swapping modularity of the VCT actuator and the EGO sensor components using network communications is presented. First, a discrete LQG controller is designed, and then this controller is distributed to the engine control unit, the VCT controller, and the EGO sensor controller in order to maximize the component swapping modularity of the system. A control oriented pre-optimization technique, which simplifies the optimization problem, and a candidate solution was devised to maximize component modularity.
\end{abstract}

Index Terms-Distributed control, engine control, mechatronic design, modularity, networked control systems, vehicle control systems.

\section{INTRODUCTION}

A LTHOUGH in the early days of computer control only one centralized computer was responsible for executing the algorithms for a particular system, increasingly, computer control algorithms reside inside individual system components in a distributed fashion. For example, there are up to 80 microcontrollers in today's high-end vehicles, and it is expected that, by $2010,90 \%$ of all computer code will reside in such embedded systems [1].

As control systems are implemented in an increasingly distributed fashion, modularity of the overall system becomes an important design decision. Ulrich and Tung [2] define modularity in terms of two characteristics of product design: 1) similarity between the physical and functional architecture of the design and 2) minimization of incidental interactions between physical components. They also state that "component-swapping modularity occurs when two or more alternative basic components can be paired with the same modular components creating different product variants belonging to the same product family".

Manuscript received June 04, 2009; revised April 08, 2010; accepted July 26, 2010. Manuscript received in final form September 17, 2010. Date of publication October 28, 2010; date of current version August 17, 2011. Recommended by Associate Editor R. Rajamani.

M. Çakmakcı is with the Department of Mechanical Engineering, Bilkent University, Ankara, Turkey, 06800 (e-mail: melihc@bilkent.edu.tr).

A. G. Ulsoy is with the Department of Mechanical Engineering, University of Michigan, Ann Arbor, MI 48109 USA (e-mail: ulsoy@umich.edu).

Color versions of one or more of the figures in this paper are available online at http://ieeexplore.ieee.org.

Digital Object Identifier 10.1109/TCST.2010.2080275
Fig. 1 describes physical and functional boundaries for a networked control system with bi-directional communications and smart components. When a component change occurs [sensor or actuator shown in Fig. 1(a)] both overall controller [Fig. 1(b)] and overall plant dynamics [Fig. 1(c)] are affected. Control systems with modularly swappable components can then be defined as systems in which the initial and final configurations due to a component change operate at their corresponding optimal performance. By using the additional design freedom, with networked control systems with bi-directional communications, it is possible to improve component swapping modularity of the system by containing the plant dynamics and corresponding control algorithm changes only in the affected components physical boundaries [3], [4].

Variable camshaft timing (VCT) is an appealing feature for automotive engines because it allows optimization of the cam timing over a wide range of operating conditions [5]. VCT schemes not only improve fuel economy [6]-[8], but also reduce emissions [9], [10] while improving full load performance [11].

VCT schemes increase internal residual gas by affecting the intake, combustion, and exhaust phases of the engine cycle. Increase in internal residual gas reduces the combustion temperature which decreases nitrogen oxide $\left(\mathrm{NO}_{\mathrm{x}}\right)$ formation. The internally recirculated exhaust gas is rich in unburned hydrocarbons, HCs, which can be burned in the next cycle under cold engine and/or large disturbance conditions. Application of VCT schemes, since they require higher manifold pressure, decrease pumping losses which results in improved fuel economy. However, dilution of the in-cylinder mixture adversely affects the engine torque response. These factors define the tradeoff between good emissions and good drivability for VCT engines.

In [12], the detailed operation of a continuous variable cam timing component is described. This VCT system works on the principle of sliding helical gears controlled by a hydraulic piston. As the helical gear moves back and forth, the rotation of the camshaft relative to the timing pulley changes (see Fig. 2, which is based on [12]).

The work presented in this paper is an extension of the method described in [3] and [4], which takes advantage of the bi-directional communication among smart components in a networked control system (NCS), to a discrete multiple-input mutiple-output (MIMO) controller applied to a higher order system. Consequences of developing modular control algorithms for automotive powertrain control systems and VCT engines have been discussed in [13]. In that work, modularity is considered at the controller level. Although the plant model presented in this paper originated from the same work (i.e., [13] and [14]), the controller used in this paper is based on the discrete linear quadratic Gaussian (LQG) design for a 
(a)

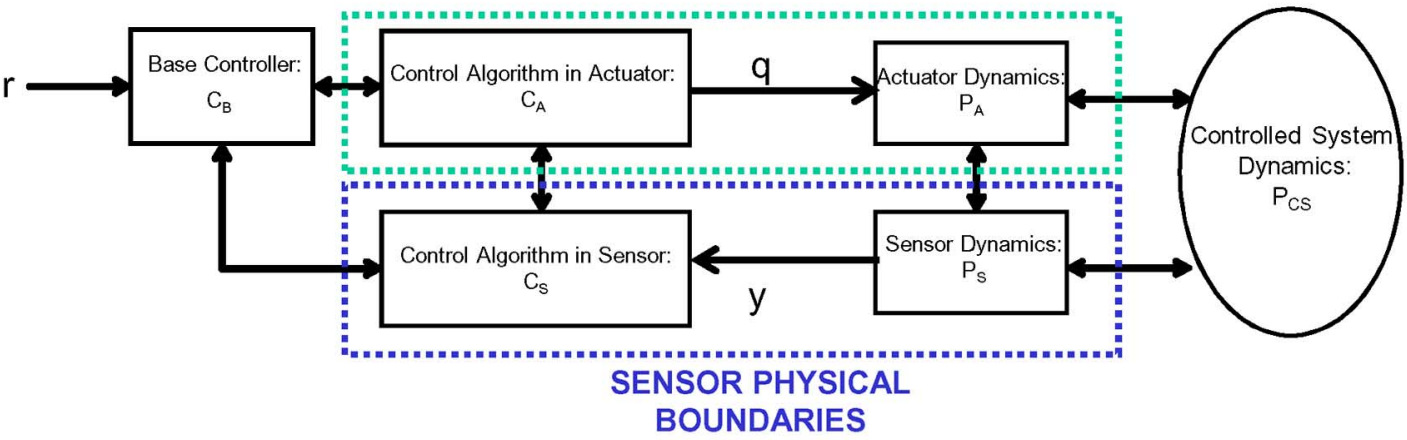

(b)

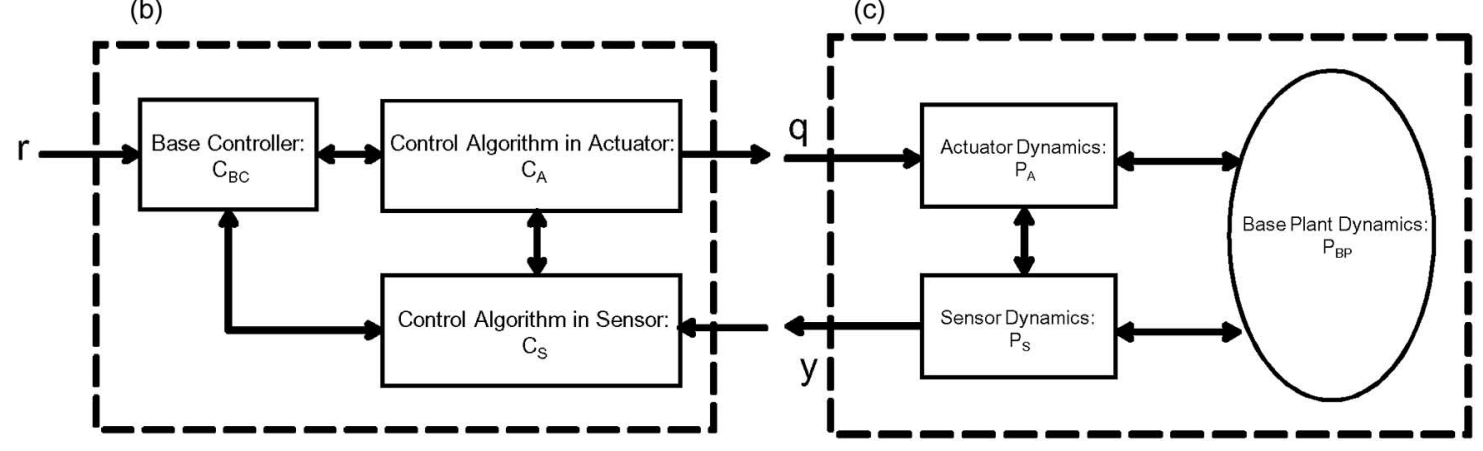

Fig. 1. Physical and functional boundaries of the control system component.

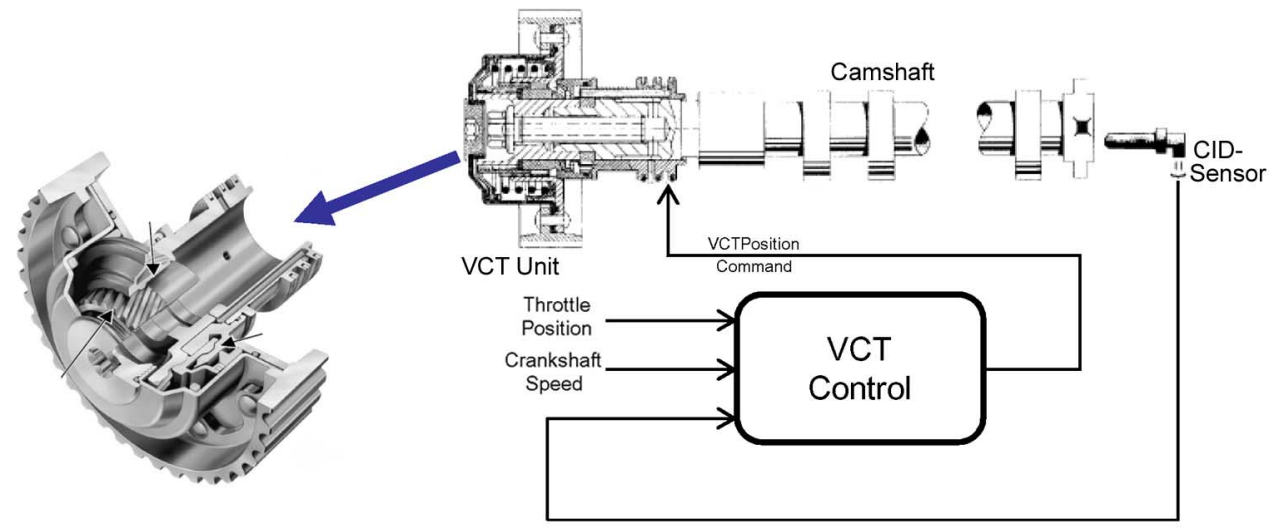

Fig. 2. Continuous VCT system.

general MIMO structure. Also, in our research, we define the module boundaries at the physical component level (i.e., the mechatronic modularity) as shown in Fig. 1(a).

In this paper, we present the design and distribution of a discrete MIMO controller for aVCT) engine. The overall controller, $\mathbf{C}$, is obtained by using a conventional control design method. Then, for the VCT and exhaust gas oxygen sensor (EGO) components separately, optimal distribution problems are solved to find the equivalent distributed controllers and the communication among them. The resulting distributed controllers maximize the component swapping modularity of the smart VCT unit and the smart EGO sensor. In Section II, the VCT engine model used in the controller design and the discrete MIMO controller design will be presented. Next, distribution of the controller design to engine control unit (ECU), VCT controller, and EGO controller to maximize component-swap- ping modularity will be discussed. Subsequently, we present our conclusions and future research plan in Section IV.

The solution presented here is obtained for a linearized version of a nonlinear model under specific operating conditions. The procedure can be repeated for multiple operating points and implemented in the real application with gain scheduling using the same controller structure found. This will improve the robustness of the controller as well as the validity of the controller obtained using the linearized model.

\section{ENGINE Modeling AND MIMO CONTROL DESIGN}

\section{A. VCT Engine Model}

The development of a continuous, nonlinear, low-frequency, phenomenological and control-oriented VCT engine model was discussed in [14] based on the model structure given in [15] and 


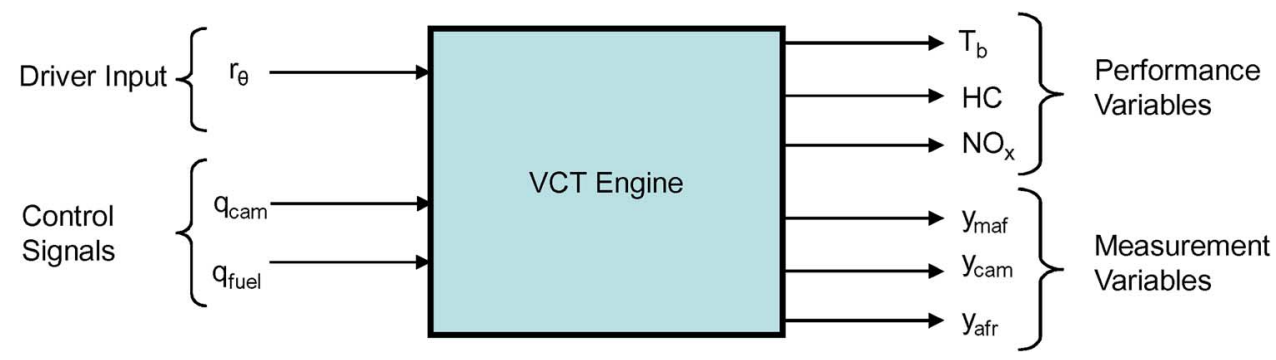

Fig. 3. I/O relationship of the dynamic plant model for control development.

other references. The input-output (I/O) relationship of the plant model developed is given in Fig. 3.

In previous work, an experimental setup was used to develop relationships for the engine breathing process, and torque generation and feed gas $\mathrm{HC}$ and $\mathrm{NO}_{\mathrm{x}}$ emissions were developed. Details of this work will not be discussed here, and the reader is referred to [14] and [5].

\section{B. Dynamics of the VCT Unit and EGO Sensor}

In order to model the VCT actuator dynamics, a first-order transfer function will be used

$$
Y_{\text {cam,actual }}(s)=\frac{-0.013 s+1 / \tau_{\mathrm{vct}, \text { act }}}{s+1 / \tau_{\mathrm{vct}, \text { act }}} Q_{\text {cam }}(s)
$$

where $\tau_{\text {vct,act }}=0.0371$ and $Q_{\text {cam }}$ is the commanded cam phase angle. The nonminimum phase zero observed in (1) is an artifact of the identification process.

For the VCT sensor, a delay of two fundamental sampling periods was assumed, modeled as a first-order Pade approximation with parameter $\tau_{\mathrm{vct}, \mathrm{sen}}$. For an $n$ cylinder engine at a speed of $N \mathrm{r} / \mathrm{min}$, the fundamental sampling rate is defined as [14]

$$
\Delta T=\frac{120}{N n} .
$$

The dynamics of the EGO sensor is modeled as first-order with a time constant $\tau_{\text {ego }}=70 \mathrm{~ms}$ given by

$$
Y_{\text {afr }}(s)=\frac{1 / \tau_{\text {ego }}}{s+1 / \tau_{\text {ego }}} Y_{\text {afr,exhaust }}(s)
$$

where $Y_{\text {afr }}$ is the measured air-fuel ratio by the EGO sensor and $Y_{\text {afr,exhaust }}$ is the actual air-fuel ratio at the exhaust assembly.

The MATLAB/SIMULINK plant model for the VCT engine was developed based on the information and regression data given in [14]. The response of the overall model was then validated using open-loop model results in [14] and [16].

\section{Discrete-Time MIMO Controller Design}

The dynamic engine model described in the previous section is linearized around the nominal inputs, i.e., CAM Angle = $10^{\circ}$, Fuel $=0$ grams, and Throttle Angle $=9.33^{\circ}$, and the corresponding steady-state internal states using MATLAB/ Simulink. The linearized model states are: the Manifold pressure, the Air-Fuel ratio (at the UEGO sensor), the Air Charge Estimator state, the Mass Air Flow, the Measured Cam Phase, the Actual Cam Phase, the Delayed Fuel, the Airfuel ratio (at the catalytic converter) and the Air-Fuel ratio (at the cylinder) [14].

The linearized model is then discretized with a sampling period $\Delta T$ to obtain

$$
\begin{aligned}
\mathbf{x}(k+1) & =\mathbf{A}_{\mathbf{d}} \mathbf{x}(k)+\mathbf{B}_{\mathbf{d}} \mathbf{u}(k)+\mathbf{B}_{\mathbf{r} \mathbf{1}, \mathbf{d}} r_{\theta}(k) \\
\mathbf{y}(k) & =\mathbf{C}_{\mathbf{d}} \mathbf{x}(k)+\mathbf{D}_{\mathbf{d}} \mathbf{u}(k)+\mathbf{B}_{\mathbf{r} \mathbf{1}, \mathbf{d} q} r_{\theta}(k)
\end{aligned}
$$

where

$$
\begin{aligned}
& \mathbf{A}_{d}=\left[\begin{array}{ll}
\mathbf{A}_{d 1} & \mathbf{A}_{d 2}
\end{array}\right] \\
& {\left[\begin{array}{lllll}
0.8984 & -0.01638 & 0.02042 & 0.0025 & 0.0003
\end{array}\right]} \\
& \mathbf{A}_{d 1}=\left[\begin{array}{ccccc}
0.8984 & -0.01638 & 0.02042 & 0.0025 & 0.0003 \\
0 & 0.8169 & 0 & 0 & 0 \\
0 & 0.1153 & 0.3679 & 0 & 0 \\
0 & -0.00107 & 0.0307 & 0.9435 & 0.022 \\
0 & 0 & 0 & 0 & 0.7575 \\
0 & 0.0045 & 0 & 0 & 0 \\
0 & 1 E-6 & -7 E-5 & 0.0002 & 1 E-5 \\
0 & -0.0238 & -0.0032 & 0.0456 & 0.0054 \\
0 & -7 E-5 & -0.0003 & 0.0005 & 5 E-5
\end{array}\right] \\
& \mathbf{A}_{d 2}=\left[\begin{array}{cccc}
0.1543 & -656.314 & -0.8944 & 10.1589 \\
0 & 0 & 0 & 0 \\
0 & 0 & 0 & 0 \\
0 & 0 & 0 & 0 \\
0 & 0 & 0 & 0 \\
0.944 & 0 & 0 & 0 \\
0 & 0.3679 & 0 & 0 \\
0.2551 & -1240 & 0.466 & -14.0665 \\
0.0011 & -6.3646 & 0.0053 & 0.9407
\end{array}\right]
\end{aligned}
$$

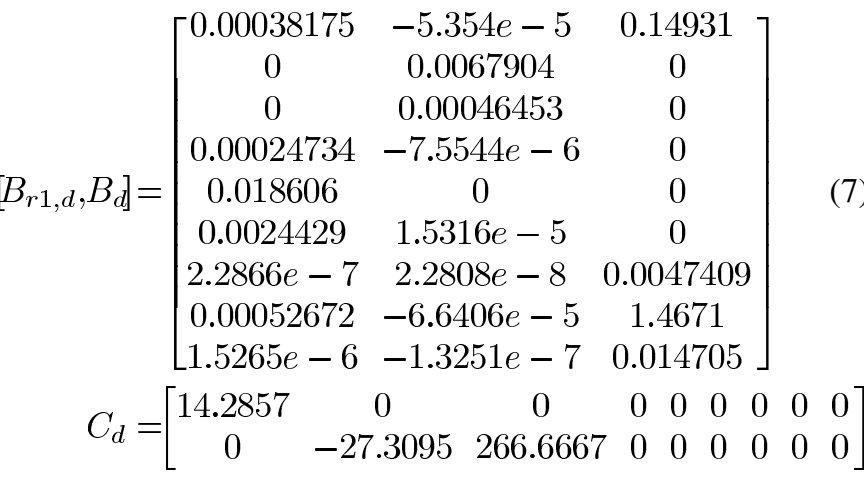

$$
\begin{aligned}
& D_{d}=\left[\begin{array}{ccc}
0 & 0 & 0 \\
0 & 0.013 & 0
\end{array}\right] \text {. }
\end{aligned}
$$

Since maintaining the stoichiometric air-fuel ratio and zero steady-state error in cam timing is important during throttle 


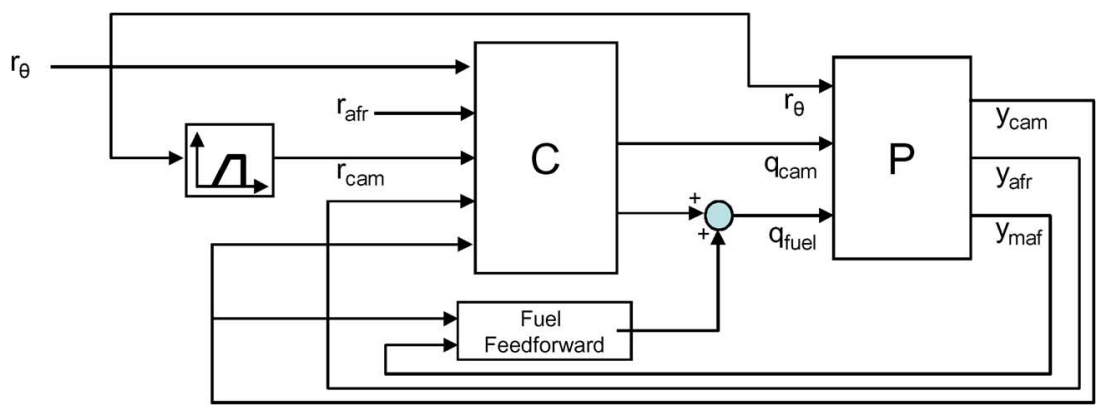

Fig. 4. VCT engine with discrete MIMO controller.
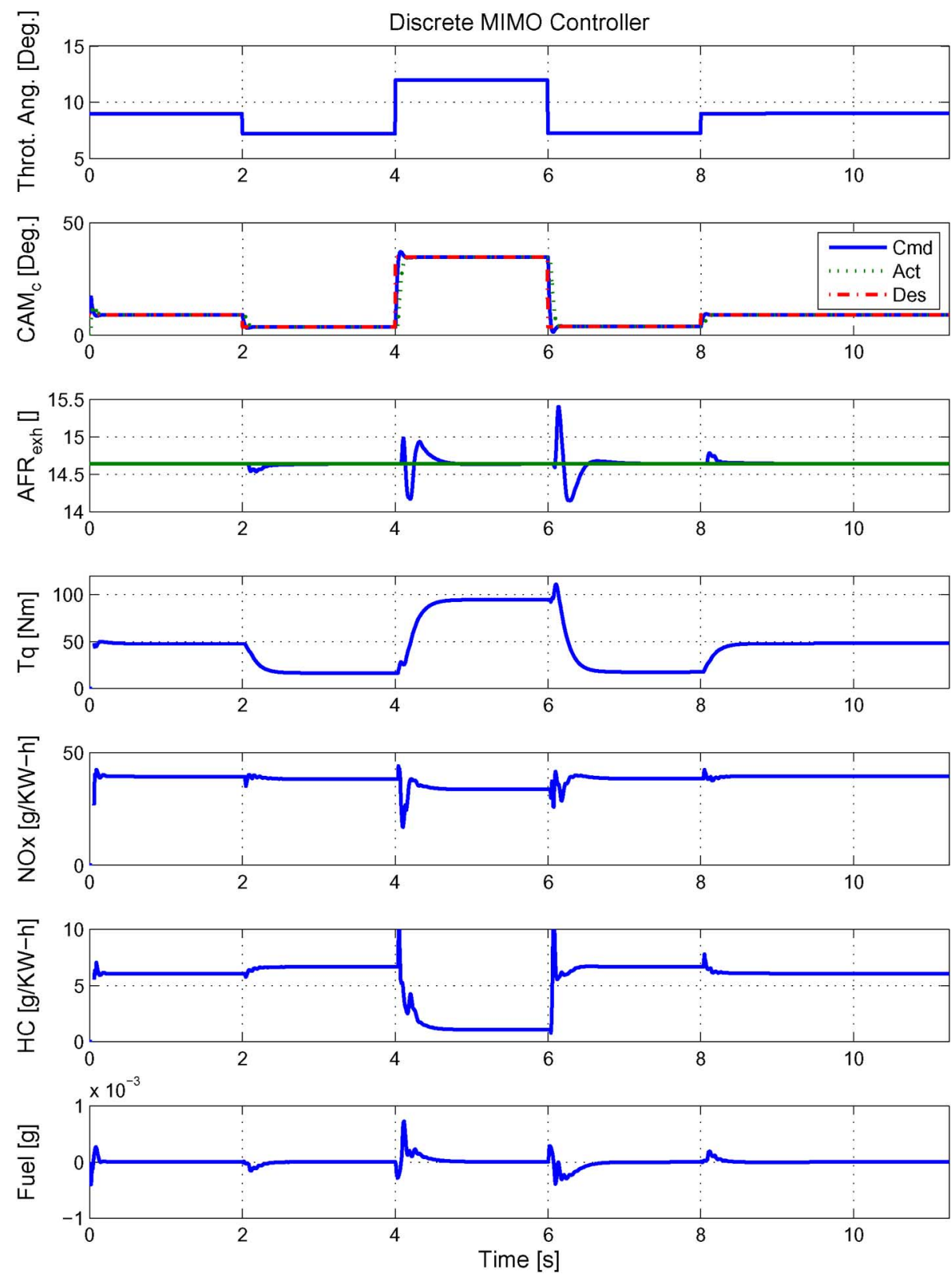

Fig. 5. VCT engine with discrete MIMO controller closed-loop response. 


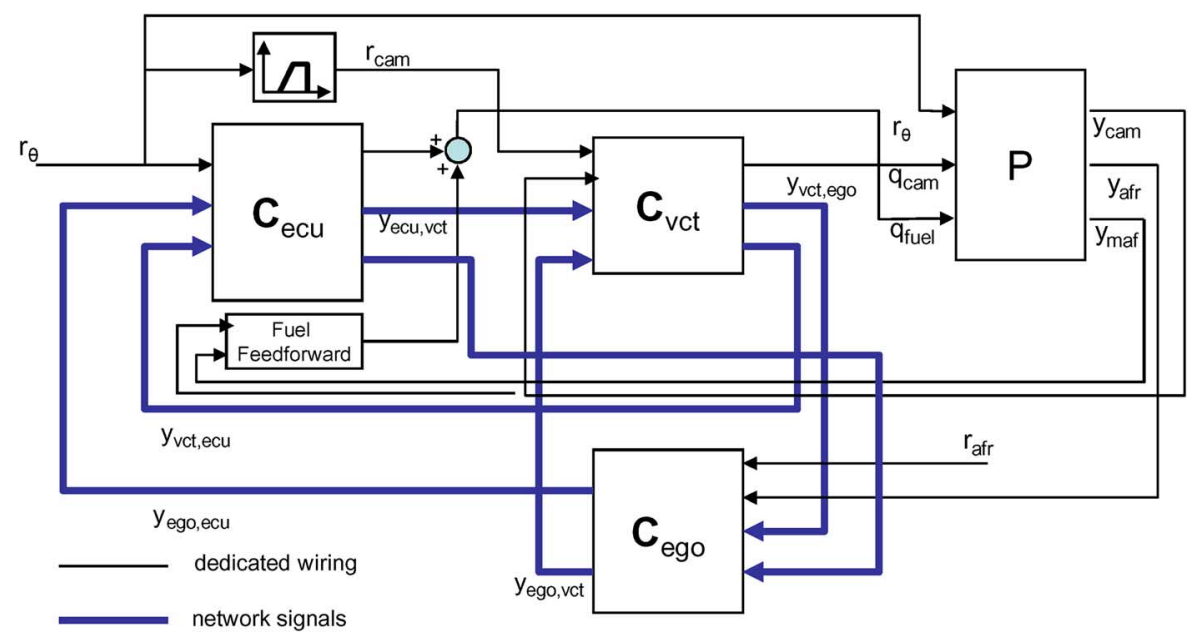

Fig. 6. VCT engine with distributed discrete MIMO controller.

angle changes, integral control of the plant outputs is implemented. This is done by augmenting the state vector with the integral of the output tracking errors

$$
\begin{array}{r}
\hat{\mathbf{x}}(k+1)=\left[\begin{array}{cc}
\mathbf{A}_{\mathbf{d}} & \mathbf{0} \\
\Delta T \mathbf{C}_{\mathbf{d}} & \mathbf{I}
\end{array}\right]\left[\begin{array}{c}
\mathbf{x}(k) \\
\mathbf{x}_{\mathbf{I}}(k)
\end{array}\right]+\left[\begin{array}{c}
\mathbf{B}_{\mathbf{d}} \\
-\Delta T \mathbf{C}_{\mathbf{d}}
\end{array}\right] \mathbf{u}(k) \\
+\left[\begin{array}{cc}
\mathbf{B}_{\mathbf{r} \mathbf{1}, \mathbf{d}} & \mathbf{0} \\
\mathbf{0} & -T_{s} \mathbf{I}
\end{array}\right]\left[\begin{array}{c}
r_{\theta}(k) \\
r_{\mathrm{cam}}(k) \\
r_{\mathrm{afr}}(k)
\end{array}\right]
\end{array}
$$

where $r_{\theta}, r_{\text {cam }}$, and $r_{\text {afr }}$ are the set points for the throttle angle, the cam phase angle, and the air-fuel ratio, respectively. By using the discrete-time linear system above an linear quadratic regulator (LQR) controller with the state feedback gains, $K_{d}$ was obtained as follows:

$$
\mathbf{K}_{d}^{T}=\left[\begin{array}{cc}
-0.00029813 & -0.013001 \\
22.2911 & 0.0006195 \\
126.643 & -0.0087846 \\
2.84 E-5 & 0.025793 \\
-2.79 E-6 & 0.0030435 \\
-0.00010603 & -0.038505 \\
1.6371 & 65.1827 \\
-0.00029203 & -0.0079145 \\
-0.065519 & -2.647 \\
0.0001704 & 0.0085496 \\
-40.1194 & 0.00095088
\end{array}\right]
$$

Since the only measurements available are the cam timing measurement and the air-fuel measurement, a Kalman filter is designed to estimate the remaining states with the gains

$$
\mathbf{L}_{d}=\left[\begin{array}{cc}
0.095152 & 8.486 e-5 \\
-9.9756 e-11 & 0.00020019 \\
-1.8683 e-11 & 0.0014151 \\
-2.6077 e-8 & 0.00012182 \\
-1.0599 e-10 & -4.7379 e-15 \\
2.8741 e-8 & 1.495 e-5 \\
-9.9113 e-7 & -2.9073 e-7 \\
-0.015256 & -6.9183 e-6 \\
0.00014419 & -1.3294 e-6
\end{array}\right]
$$

The closed-loop system with the discrete MIMO controller is given in Fig. 4. The resulting closed-loop (i.e., discrete controller + nonlinear plant model) response to a throttle profile is shown in Fig. 5.

\section{DistRIBUtion PROBLEM}

The block diagram representing the plant and controller relationship for the discrete-time MIMO controller in the previous section is given in Fig. 4. With the controller distribution problem, our aim is to find component controllers, $\mathbf{C}_{\mathrm{ecu}}, \mathbf{C}_{\mathrm{vct}}$, $\mathrm{C}_{\text {ego }}$, that improve the component-swapping modularity of the system by utilizing the bi-directional network communications. The block diagram of the proposed distributed system with the proposed communication is given in Fig. 6 .

A generic optimization problem formulation for maximizing the component-swapping modularity of an actuator component was given in [17]. Given nominal settings for the plant parameters (denoted as $\mathbf{p}_{\mathrm{CS}}^{0}, \mathbf{p}_{\mathrm{VCT}}^{0}$, and $\mathbf{p}_{\mathrm{EGO}}^{0}$ for the controlled system, i.e., rest of the engine, VCT component, and EGO sensor, respectively), we can formulate the distribution problem that maximizes VCT component-swapping modularity $M_{\mathrm{VCT}}$ while the distribution constraint, which is that the desired overall controller must be equal to the overall effect of the distributed controller (i.e., $C_{\mathrm{des}}=C_{\mathrm{dis}}$ ), holds.

In short, $M_{\mathrm{VCT}}$ represents the size of the region in the parameter space, which includes the nominal VCT parameters, for which we can ensure the distribution constraint by changing only gains of the VCT controller.

\section{A. Formulation of $\mathbf{C}_{\mathrm{des}}$}

Here, the formulation for the desired centralized controller $C_{\text {des }}$ given plant parameters $\mathbf{p}_{\mathrm{CS}}^{0}, \mathbf{p}_{\mathrm{VCT}}^{0}$, and $\mathbf{p}_{\mathrm{EGO}}^{0}$ is presented. Given the optimal state feedback matrix $\mathbf{K}_{d}=\left[\mathbf{K} \mathbf{1}_{d}, \mathbf{K} \mathbf{2}_{d}\right]$ and linear observer gain $\mathbf{L}_{d}$, the state 

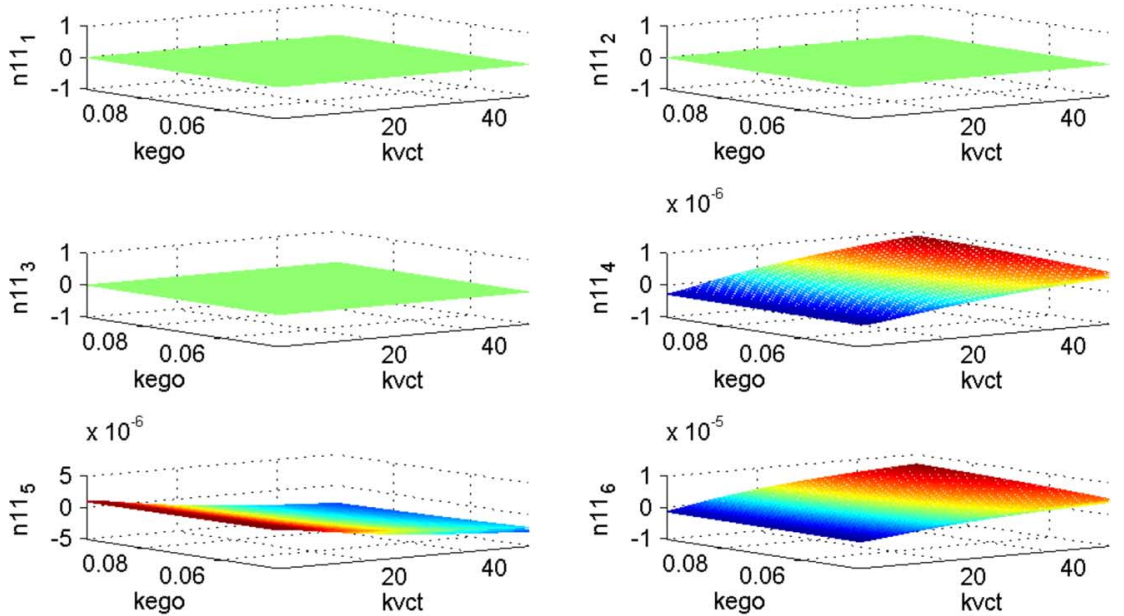

$\times 10^{-5}$
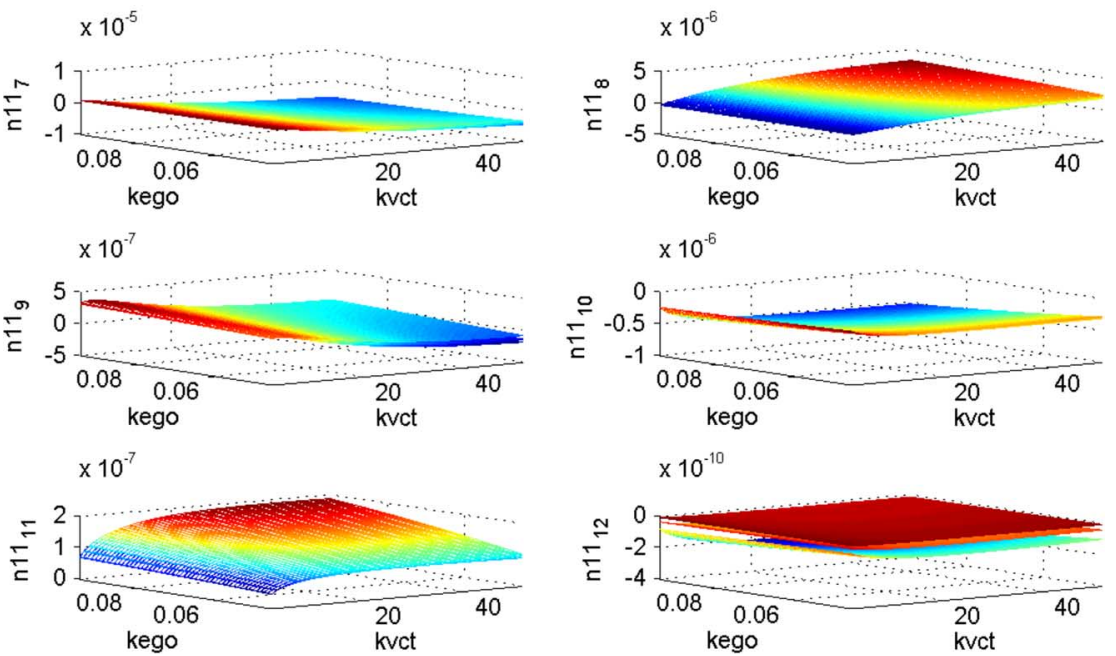

Fig. 7. Polynomial coefficients for $N_{11}(z)$ as $\boldsymbol{\tau}_{\text {vct }}$ and $\tau_{\text {ego }}$ are varied.

space representation of the discrete MIMO controller in Fig. 4 can be given as

$$
\begin{aligned}
\hat{\mathbf{x}}_{c}(k+1) & =\mathbf{A} \mathbf{c}_{d}\left[\begin{array}{c}
\mathbf{x}_{c} \\
\mathbf{x}_{c, I}
\end{array}\right](k)+\mathbf{B} \mathbf{c}_{d} \mathbf{u}(k)+\mathbf{B} \mathbf{c}_{\mathbf{d}, \mathbf{r}}\left[\begin{array}{c}
r_{\theta} \\
r_{\text {cam }} \\
r_{\text {afr }}
\end{array}\right] \\
\mathbf{q}(k) & =\mathbf{C}_{d}\left[\begin{array}{c}
\mathbf{x}_{c} \\
\mathbf{x}_{c_{I}}
\end{array}\right](k)
\end{aligned}
$$

where $\mathbf{A} \mathbf{c}_{\mathbf{d}}$ is given by (15), shown at the bottom of this page, and

$$
\begin{aligned}
\mathbf{B} \mathbf{c}_{\mathbf{d}} & =\left[\begin{array}{c}
\mathbf{L}_{\mathbf{d}} \\
-T_{s} \mathbf{I}
\end{array}\right] \\
\mathbf{B} \mathbf{c}_{\mathbf{d}, \mathbf{r}} & =\left[\begin{array}{cc}
\mathbf{B} \mathbf{r}_{\mathbf{d}}-\mathbf{L}_{\mathbf{d}} \mathbf{D r}_{\mathbf{d}} & \mathbf{0} \\
\mathbf{0} & T_{s} \mathbf{I}
\end{array}\right]
\end{aligned}
$$
the form

$$
\mathbf{C}=\left[\begin{array}{lll}
N_{11}(z) / D_{11}(z) & \cdots & N_{15}(z) / D_{15}(z) \\
N_{21}(z) / D_{21}(z) & \cdots & N_{25}(z) / D_{25}(z)
\end{array}\right]
$$

$N_{11}$ and $D_{11}$ are polynomials with vectors $\mathbf{x}_{n 11}$ and $\mathbf{x}_{d 11}$ of controller gains, respectively, given by

$$
\begin{aligned}
& C_{11}\left(\mathbf{x}_{\mathbf{n} 11}, \mathbf{x}_{\mathbf{d} 11}, z\right) \\
& \quad=\frac{N_{11}\left(\mathbf{x}_{\mathbf{n} 11}, z\right)}{D_{11}\left(\mathbf{x}_{\mathbf{d} 11}, z\right)} \\
& \quad=\frac{x_{n 11, m} z^{m+1}+\ldots+x_{n 11, n+2} z+x_{n 11, n+1}}{x_{d 11, n} z^{n+1}+\ldots+x_{d 11,2} z+x_{d 11,1}} .
\end{aligned}
$$

$$
A c_{d}=\left[\begin{array}{cc}
A_{d}-L_{d} C_{d}-B_{d} K 1_{d}+L_{d} D_{d} K 1_{d} & \mathbf{L}_{d} D_{d} K 2_{d}-B_{d} K 2_{d} \\
0 & I
\end{array}\right]
$$




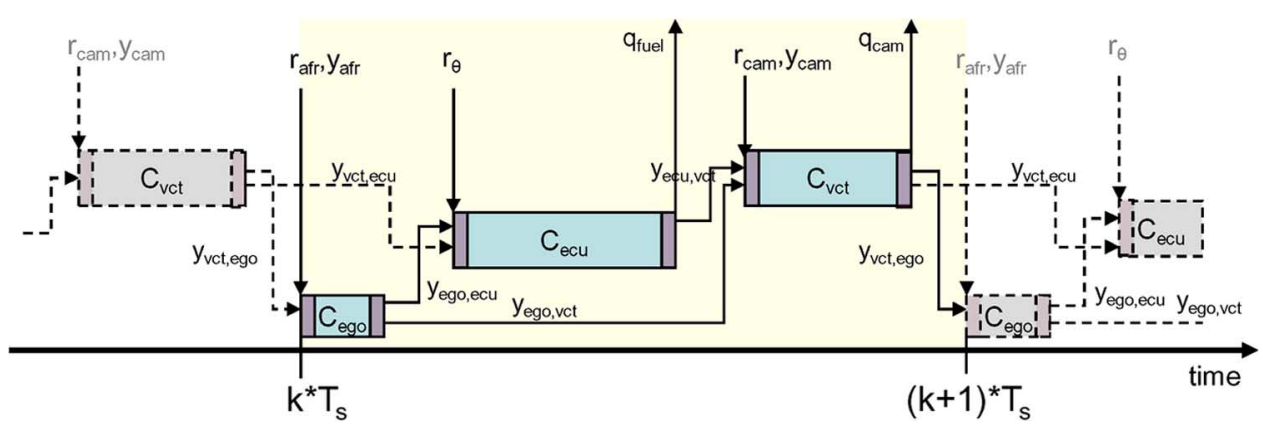

Fig. 8. Real-time schedule for distributed discrete MIMO controller.

Then, we can define $C_{\mathrm{des}}\left(\mathbf{p}_{\mathrm{VCT}}, \mathbf{p}_{\mathrm{EGO}}, \mathbf{p}_{\mathrm{ECU}}\right)$ as

$$
\begin{aligned}
& C_{\mathrm{des}}\left(\mathbf{p}_{\mathrm{VCT}}, \mathbf{p}_{\mathrm{EGO}}, \mathbf{p}_{\mathrm{CS}}\right) \\
& \quad=C\left(\left\{\mathbf{x}_{n i j}^{*}, \mathbf{x}_{d i j}^{*} \mid i=1,2 ; j=1,2, \ldots, 5\right\}\right)
\end{aligned}
$$

where $\left\{\mathbf{x}_{n i j}^{*}, \mathbf{x}_{d i j}^{*} \mid i=1,2 ; j=1,2, \ldots, 5\right\}=\operatorname{argmin}$ $J_{C}(P, \mathbf{C})=\Sigma_{1}^{\infty}\left(x(k)^{\prime} Q x(k)+u(k)^{\prime} R u(k)\right)$ s.t. $\mathbf{x}(k+1)=$ $\mathbf{A}_{d} \mathbf{x}(k)+\mathbf{B}_{d} \mathbf{u}(k)$. $\mathbf{p}_{\mathrm{CS}}, \mathbf{p}_{\mathrm{VCT}}$, and $\mathbf{p}_{\mathrm{EGO}}$ are parameter vectors representing the controlled system (rest of the engine), VCT component, and EGO sensor parameters, respectively.

These polynomial coefficients vary as VCT and EGO plant parameters change, as shown in Fig. 7 for $N_{11}(z)$, and can be implemented in the numerical solution phase of the problem as lookup tables or regression equations.

\section{B. Formulation of $\mathbf{C}_{\mathrm{dist}}$}

For the control system given in Fig. 6 and real-time distributed controller schedule given in Fig. 8, discrete MIMO equations to calculate the overall controller equation can be written to obtain the equation given in (22), shown at the bottom of this page.

It is also important to note that Fig. 8 assumes perfect completion of the communication scheme and the effect of communication delays and bandwidth limitations are not considered while finding the equivalent controller given in (22). However, these effects could be investigated in future work by including delays on various communication paths when such information is available.

In order to obtain the distribution constraint needed to solve the distribution problem numerically, we need to make assumptions regarding the order of polynomial transfer function matrices $\mathbf{C}_{\text {ecu }}, \mathbf{C}_{\mathrm{vct}}$, and $\mathbf{C}_{\text {ego }}$. Although the most straightforward approach would be to assume all $\mathbf{C}_{\mathrm{ecu}}, \mathbf{C}_{\mathrm{vct}}$, and $\mathbf{C}_{\text {ego }}$ are composed of transfer functions of the same order of the centralized controller $\mathbf{C}_{\text {des }}$, this may not be preferable, since it increases the numerical burden of the problem substantially. For the application we are considering, here our desired controller transfer matrix consists of polynomials of 11th order, which means our nonlinear optimization problem would have almost 600 design variables and constraints to handle.

A better way to tackle the numerical burden of the distribution problem is to perform some pre-optimization analysis to better understand and simplify the problem. Fig. 9 shows the pole zero map of the desired optimal controller $\mathbf{C}_{\mathrm{des}}$ obtained in the previous section as the VCT component parameters $\tau_{\mathrm{vct}, \text { act }}$ and $\tau_{\mathrm{vct}, \text { sen }}$ vary.

Analyzing these plots carefully, we note that swapping the VCT component with another one would only effect the elements $C_{11}, C_{12}, C_{13}, C_{14}, C_{15}, C_{23}, C_{25}$ (due to varying poles, zeros or both in Fig. 9) of our desired optimal controller, $\mathbf{C}_{\mathrm{des}}$. Even in these elements, one can observe some stationery poles and zeros as well as varying poles and zeros which can be exploited for the purposes of the improving modularity the system.

$$
\begin{aligned}
& \mathbf{C}_{\text {dist }}(z)=-\left[\begin{array}{cccccc}
C_{\mathrm{vct} 13} & 0 & 0 & 0 & 0 & C_{\mathrm{vct} 14} \\
0 & 0 & 0 & C_{\text {ecu13 }} z^{-1} & C_{\text {ecu12 }} & 0
\end{array}\right]\left[\begin{array}{cccccc}
-1 & 0 & 0 & C_{\text {ecu23 } 3 / z} & C_{\text {ecu22 }} & 0 \\
0 & -1 & 0 & C_{\text {ecu33 }} / z & C_{\text {ecu32 }} & 0 \\
C_{\text {vct23 }} & 0 & -1 & 0 & 0 & C_{\text {vct24 }} \\
C_{\text {vct33 }} & 0 & 0 & -1 & 0 & C_{\mathrm{vct} 34} \\
0 & C_{\text {ego14 }} / z & C_{\text {ego13 }} / z & 0 & -1 & 0 \\
0 & C_{\text {ego24 }} / z & C_{\text {ego23 }} / z & 0 & 0 & -1
\end{array}\right]^{-1} \\
& \times\left[\begin{array}{ccccc}
C_{\text {ecu21 }} & 0 & 0 & 0 & 0 \\
C_{\text {ecu31 }} & 0 & 0 & 0 & 0 \\
0 & 0 & C_{\text {vct21 }} & 0 & C_{\text {vct22 }} \\
0 & 0 & C_{\text {vct31 }} & 0 & C_{\text {vct32 }} \\
0 & C_{\text {ego11 }} & 0 & C_{\text {ego12 }} & 0 \\
0 & C_{\text {ego21 }} & 0 & C_{\text {ego21 }} & 0
\end{array}\right]+\left[\begin{array}{ccccc}
0 & 0 & C_{\text {vct11 }} & 0 & C_{\text {vct12 }} \\
C_{\text {ecu11 }} & 0 & 0 & 0 & 0
\end{array}\right]
\end{aligned}
$$




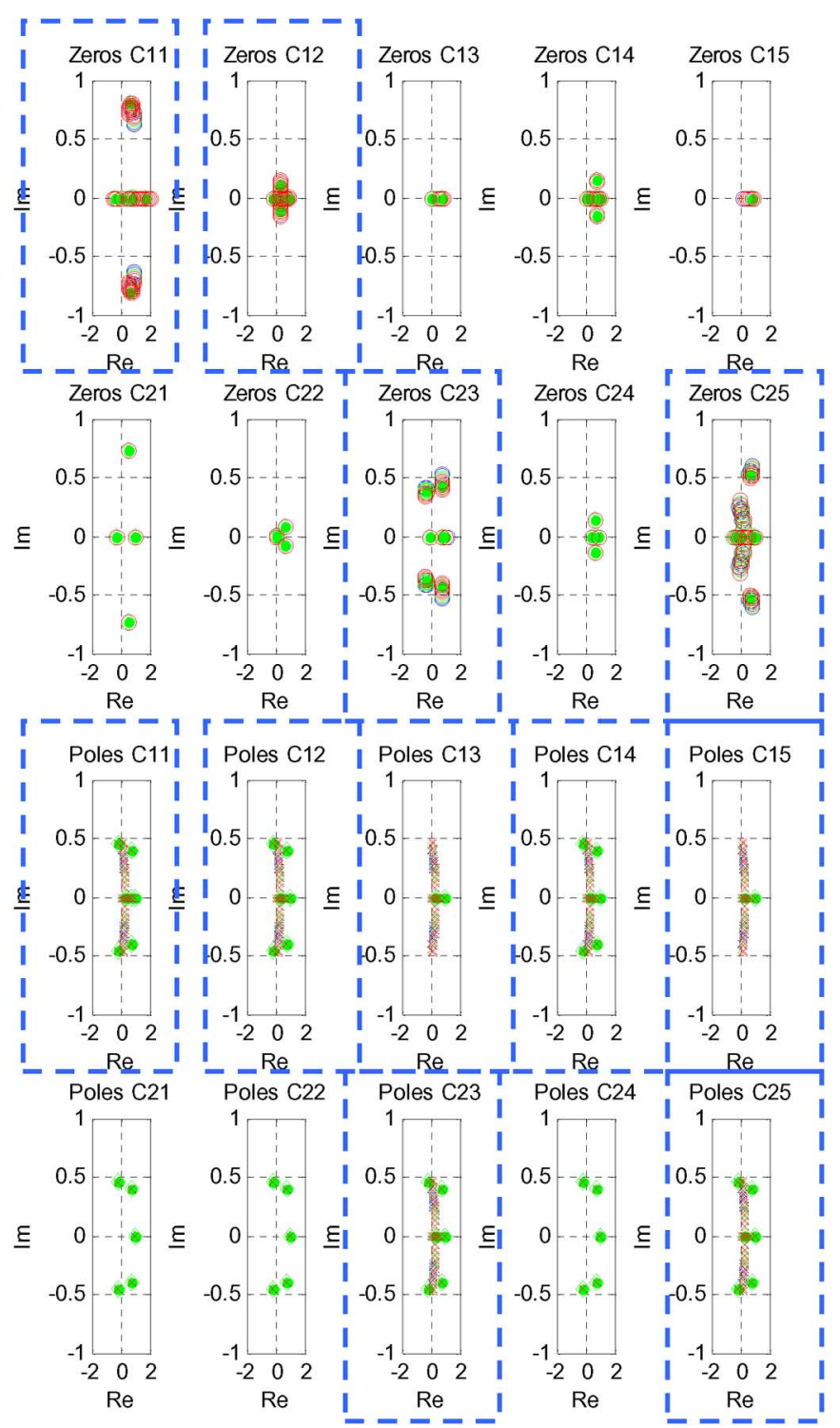

Fig. 9. Pole zero map of the $\mathbf{C}_{\mathrm{des}}(z)$ as the VCT component (i.e., $\tau_{\mathrm{vct}}$,act and $\left.\tau_{\mathrm{vct}, \text { sen }}\right)$ changes. Pole or zero plots with the dashed boxes are assumed to vary as VCT component changes.

Fig. 10 summarizes the common pole zero location variations we have observed as plant parameters vary. The static pole and zero elements can be placed in the ECU and EGO sensor controller while varying poles can be strategically placed in the VCT Controller. Pole zero cancellations due to transformation from state space to transfer function formulations further reduce the order of the controller matrix.

In order to simplify the numerical optimization problem and obtain a good candidate distribution solution to start

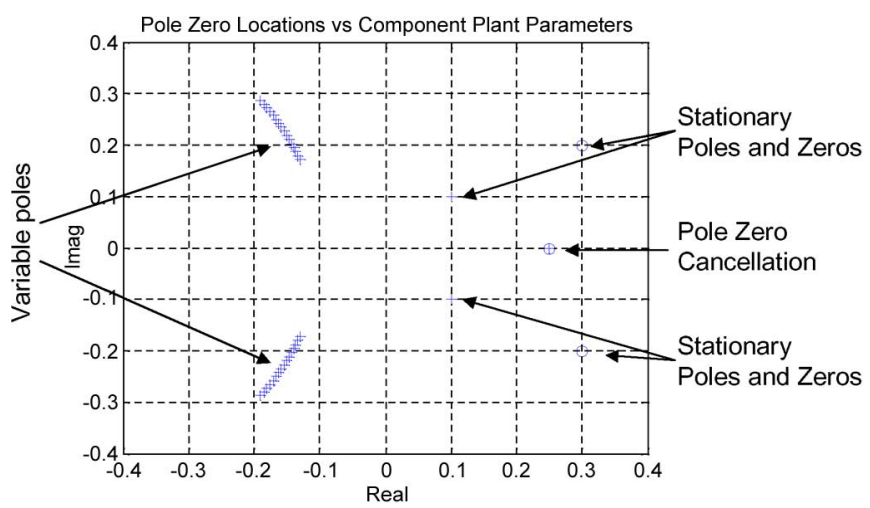

Fig. 10. Simple pole zero map of the $\mathbf{C}_{\text {des }}(z)$ as plant variables change.

the search algorithm, we used a two-step configuration design process, given below, once the pole-zero mapping of $\mathbf{C}_{\text {des }}\left(\mathbf{p}_{\mathrm{cs}}, \mathbf{p}_{\mathrm{vct}}, \mathbf{p}_{\text {ego }}, z\right)$ elements versus the VCT component plant variables $\mathbf{p}_{\mathrm{vct}}$ is obtained.

Step 1) Identify proper transfer functions $C_{\text {des,ij }}^{s}(z)$ and $C_{\mathrm{des}, \mathrm{ij}}^{v}(z)$ such that $C_{\mathrm{des}, \mathrm{ij}}(z)=$ $C_{\mathrm{des}, \mathrm{ij}}^{s}(z) C_{\mathrm{des}, \mathrm{ij}}^{v}(z)$.

Step 2) Find $\mathbf{C}_{\text {ecu }}(z), \mathbf{C}_{\text {vct }}(z)$ (with the $k$ th-row $l$ th-column element denoted as $\left.C_{\mathrm{vct}, \mathrm{kl}}(z)\right)$ and $\mathbf{C}_{\text {ego }}(z)$ satisfying (a) and (b).

a) $\mathbf{C}_{\text {dist }}\left(\mathbf{C}_{\text {ecu }}, \mathbf{C}_{\text {vct }}, \mathbf{C}_{\text {ego }}, z\right)$ is equal to $\mathbf{C}_{\mathrm{des}}\left(\mathbf{p}_{\mathrm{cs}}, \mathbf{p}_{\mathrm{vct}}, \mathbf{p}_{\mathrm{ego}}, z\right)$.

b) For all $C_{\mathrm{des}, \mathrm{ij}}^{v}(z)$ [identified in Step 2)], there exists $C_{\mathrm{vct}, \mathrm{kl}}(z)$ such that $C_{\mathrm{dist}, \mathrm{ij}}=$ $C_{\mathrm{des}, \mathrm{ij}}^{s}(z) C_{\mathrm{vct}, \mathrm{kl}}(z)$. Therefore, $C_{\mathrm{vct}, \mathrm{kl}}(z)$ can be picked to be $C_{\mathrm{des}, \mathrm{ij}}^{v}(z)$ for different VCT components.

The superscript " $s$ " denotes the stationary pole/zero pairs that do not change as $\tau_{\mathrm{vct}, \text { act }}$ and/or $\tau_{\mathrm{vct}, \text { sen }}$ change while the superscript " $v$ " denotes those that do vary. It is important to note that the primary goal of this procedure is to place varying poles and zeros to the target component controller while satisfying the distribution constraint in step 2a). Therefore, it is still acceptable if there are cases where some or all of the stationary poles/zeros reside to satisfy the distribution constraint in step 2a).

The pre-optimization method described above is generic. However, the results (i.e., number of pole zero cancellations, identifying stationary transfer function matrix elements and/or poles and zeros in these elements) would change from one application to another.

Based on the procedure given above, we obtained a candidate solution that maximizes VCT component modularity as shown in Fig. 11. Implementation of this distribution would result in an overall controller given, in (23), shown at the bottom of this page.

$$
\mathbf{C}_{\text {dist }}=\left[\begin{array}{ccccc}
C_{\mathrm{vct} 13} C_{\mathrm{ecu} 21} & C_{\mathrm{vct} 14} C_{\mathrm{ego} 21} & C_{\mathrm{vct} 11} & C_{\mathrm{vct} 14} C_{\mathrm{ego} 22} & C_{\mathrm{vct} 12} \\
C_{\mathrm{ecu} 11} & C_{\mathrm{ecu} 12} C_{\mathrm{ego} 11} & C_{\mathrm{ecu} 13} C_{\mathrm{vct} 31} / z & C_{\mathrm{ecu} 12} C_{\mathrm{ego} 12} & C_{\mathrm{ecu} 13} C_{\mathrm{vct} 32} / z
\end{array}\right]
$$




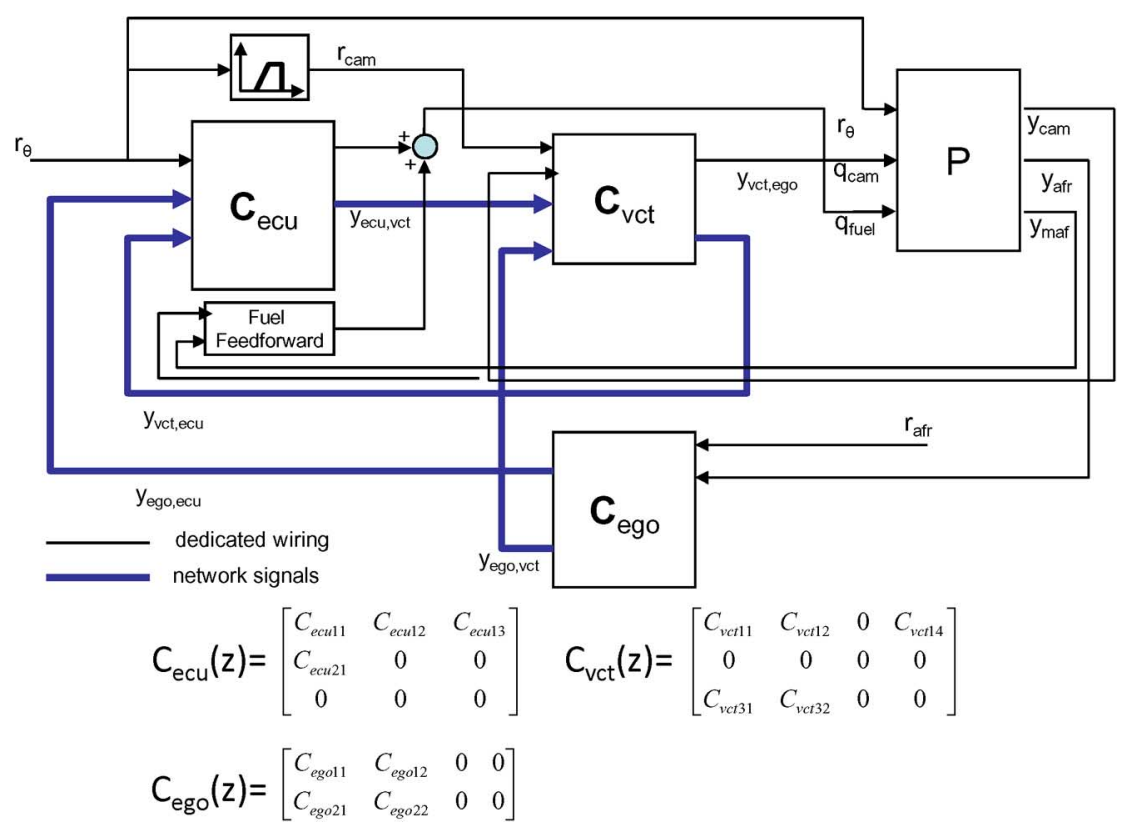

Fig. 11. Candidate distribution to maximize VCT modularity.

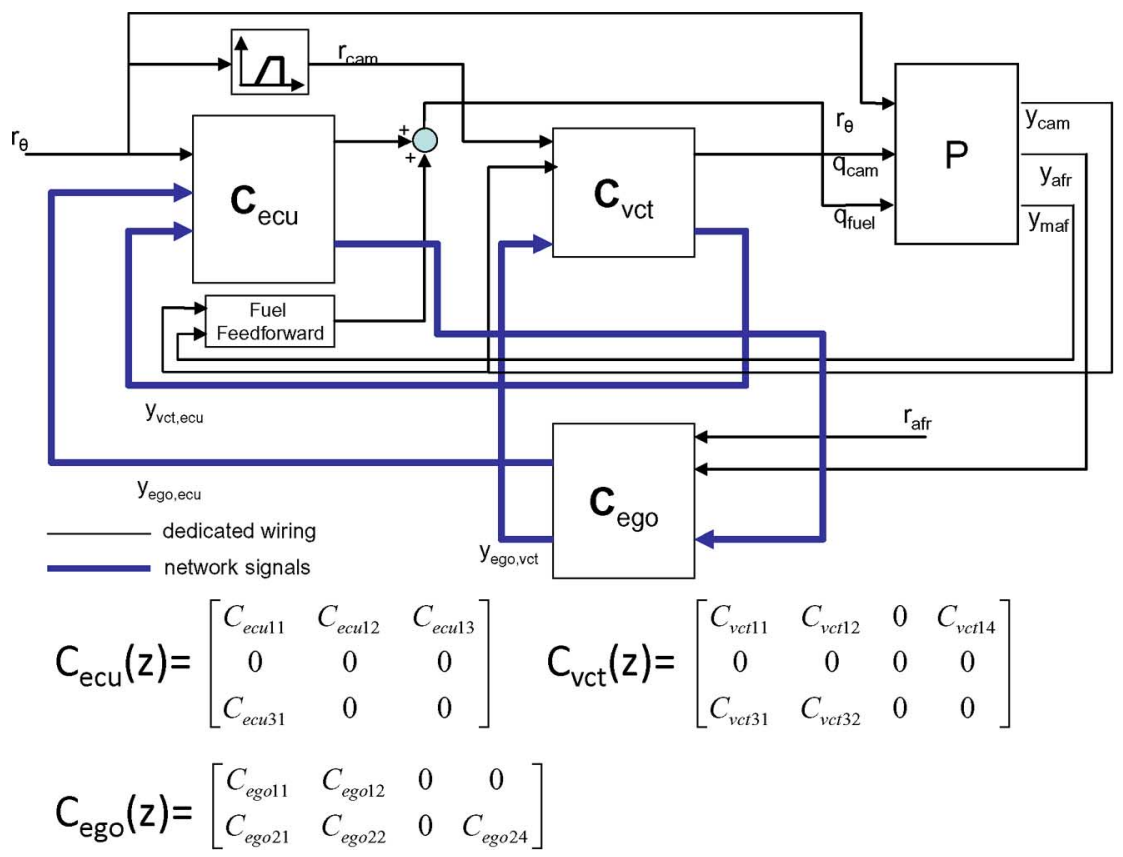

Fig. 12. Candidate distribution to maximize EGO modularity.

The closed-loop response of this solution is virtually indistinguishable from the overall controller response presented in Fig. 5. The solution for the optimization problem to maximize VCT component modularity (i.e., parameters for $\mathbf{C}_{\mathrm{ecu}}, \mathbf{C}_{\mathrm{vct}}$, and $\mathbf{C}_{\text {ego }}$ transfer function matrices) is the same as the candidate solution as presented in Table I. The numerical value of $M_{\mathrm{VCT}}^{*}$ is $60 * 10=600(\mathrm{~ms})^{2}$. A better explanation of this number can be given as follows: Assuming the default configuration for other components, optimal controllers can be obtained by only adjusting VCT component controller for VCT plant parameters changing in the ranges $\tau_{\mathrm{vct}, \text { act }}=[7,67] \mathrm{ms}$ and $\tau_{\mathrm{vct}, \mathrm{sen}}=[10,20] \mathrm{ms}$.
The same steps can also be followed to obtain a candidate solution to maximize EGO sensor modularity: After a similar pole/zero analysis to the one shown in Fig. 9, we have identified only $C_{11}$ and $C_{12}$ elements having varying elements as the EGO sensor changes. By applying the pre-optimization procedure, the candidate solution given in Fig. 12 is obtained. As in the VCT case, the closed-loop response of this solution is virtually indistinguishable from the overall controller response presented in Fig. 5. Since our static pole/zero numerical threshold is within the distribution constraint limits in the optimization problem, our candidate solution (Fig. 12 and Table II) is verified as the optimal distribution which results in $M_{\mathrm{EGO}}^{*}=60 \mathrm{~ms}$ 
TABLE I

TRANSFER FUNCTIONS FOR THE MODULAR VCT CONTROLLER SOLUTION

\begin{tabular}{|l|c||l|c|}
\hline Element & Solution & Element & Solution \\
\hline$C_{\text {ecu } 11}$ & $C_{21}$ & $C_{\text {ecu } 12}$ & 1 \\
$C_{\text {ecu } 13}$ & $C_{23}^{s} / z$ & $C_{\text {ecu } 21}$ & $C_{11}^{s}$ \\
$C_{\text {vct } 11}$ & $C_{13}$ & $C_{\text {vct } 12}$ & $C_{15}$ \\
$C_{v c t 13}$ & $C_{11}^{v}$ & $C_{\text {vct } 14}$ & $C_{14}^{v}$ \\
$C_{\text {vct } 31}$ & $C_{23}^{v}$ & $C_{\text {vct } 32}$ & $C_{25}^{v}$ \\
$C_{\text {ego } 11}$ & $C_{22}$ & $C_{\text {ego } 12}$ & $C_{24}$ \\
$C_{\text {ego } 21}$ & $C_{12}^{s}$ & $C_{\text {ego } 22}$ & $C_{14}^{s}$ \\
\hline
\end{tabular}

TABLE II

TRANSFER FUNCTIONS FOR THE MODULAR EGO CONTROLLER SOLUTION

\begin{tabular}{|l|c||l|c|}
\hline Element & Solution & Element & Solution \\
\hline$C_{\text {ecu } 11}$ & $C_{21}$ & $C_{\text {ecu } 12}$ & 1 \\
$C_{\text {ecu } 13}$ & 1 & $C_{\text {ecu } 31}$ & $C_{11}^{s}$ \\
$C_{\text {vct } 11}$ & $C_{13}$ & $C_{\text {vct } 12}$ & $C_{15}$ \\
$C_{\text {vct } 14}$ & 1 & $C_{\text {vct } 31}$ & $C_{23} z$ \\
$C_{\text {vct } 32}$ & $C_{25} z$ & $C_{\text {ego } 11}$ & $C_{22}$ \\
$C_{\text {ego } 13}$ & $C_{24}$ & $C_{\text {ego } 21}$ & $C_{12}^{s} C_{12}^{v}$ \\
$C_{\text {ego } 22}$ & $C_{14}$ & $C_{\text {ego } 24}$ & $C_{11}^{d} z$ \\
\hline
\end{tabular}

(i.e., given the default configuration, optimal controllers can be obtained by only changing EGO controller in the range $\tau_{\mathrm{EGO}}=$ $[40,100] \mathrm{ms})$.

\section{CONCLUSION}

In this paper, a method to distribute the discrete MIMO controller for the VCT engine to improve the component-swapping modularity of the VCT actuator or the EGO sensor components is presented. This work is an extension of the method described in [3] and [4] to a discrete MIMO controller applied to a more complex system. In Section II-C, a discrete LQG controller was designed based on [14]. In Section III-B, this controller was distributed to an ECU, a VCT controller, and an EGO sensor controller in order to improve the component-swapping modularity of the system. In Section III-B, we also present a control-oriented pre-optimization technique which simplifies the optimization problem, which results in shorter computation times to obtain a solution. The pre-optimization method identifies and groups together controller poles/zeros which change, or remain unchanged, as the VCT or EGO parameters vary. A candidate solution was devised and used in the optimization problem as the initial solution. Resulting solutions maximizing VCT component modularity and EGO sensor modularity are given in Figs. 11 and 12, respectively. The performances of these distributed controllers are identical to the performance of the original controller, as shown in Fig. 5. The range of the obtained solutions depends on stability and properness of the overall controller and distributed controllers as well as the optimization toleration settings.

These distributed controllers provide component-swapping modularity that does not exist in the case of implementing the whole algorithm using a single centralized controller (i.e., using the ECU only) without losing the optimal closed-loop performance. In the case of the VCT component, assuming the default configuration for other components and using the new distributed structure, optimal controllers can be obtained by only adjusting the VCT component controller for VCT plant parameters changing in the ranges $\tau_{\mathrm{vct}, \text { act }}=[7,67] \mathrm{ms}$ and $\tau_{\mathrm{vct}, \mathrm{sen}}=[10,20] \mathrm{ms}$. For the case of the EGO sensor with the same assumptions, optimal controllers can be obtained by only changing the EGO controller in the range $\left.\tau_{\mathrm{EGO}}=[40,100] \mathrm{ms}\right)$. The MIMO controller was designed using a linearized model around a specific operating point. It is expected that the practical implementation of this controller would be done by designing the MIMO controller for various operating points of the VCT engine and then using gain scheduling to calibrate the controller for different operating points. The same schedule-oriented approach can be applied to the distributed controller by solving the distribution problem for all of the operating points considered. The minimum component-swapping modularity obtained among operating points would then be used for optimization problem purposes.

Future work on this topic will include consideration of VCT and EGO modularity concurrently and improving our solution methodology such as making use of the LPV methods and/or LMIs.

\section{REFERENCES}

[1] K. J. Astrom, "Present developments in control applications," presented at the IFAC 50th Anniversary Meeting, Heidelberg, Germany, 2006, Keynote Paper.

[2] K. Ulrich and K. Tung, "Fundamentals of product modularity," in Issues in Mechanical Design International, A. Sharon, Ed. New York: ASME, 1991, pp. 73-79.

[3] M. Cakmakci and A. G. Ulsoy, "Networked control systems with bi-directional communication among "smart" components," in Proc. Amer. Control Conf., Jun. 2005, pp. 627-632.

[4] M. Cakmakci and A. G. Ulsoy, "Improving component-swapping modularity using bidirectional communication in networked control systems," IEEE/ASME Trans. Mechatron., vol. 14, no. 3, pp. 307-316, Jun. 2009.

[5] A. Stefanopoulou, J. Cook, J. Grizzle, and J. Freudenberg, "Controloriented model of a dual equal variable cam timing spark ignition engine," ASME J. Dynam. Syst, Meas. Control, vol. 120, pp. 257-266, 1998.

[6] A. C. Elrod and M. T. Nelson, "Development of variable valve timing engine to eliminate the pumping losses associated with throttled operation," SAE Paper No. 860537, 1986.

[7] C. Gray, "A review of variable engine valve timing," SAE Transactions, vol. 97, pp. 631-641, 1989.

[8] T. Ma, "Effect of variable engine valve timing on fuel economy," in Proc. Soc. Automotive Eng. Int. Congress Expo., 1988, vol. 29, pp. $665-672$.

[9] G. Meacham, "Variable cam timing as an emission control tool," 1970, SAE Paper No. 700645.

[10] R. Stein, K. Galietti, and T. Leone, "Dual equal Vct-A variable camshaft timing strategy for improved fuel economy and emissions," SAE Paper No. 950975, 1995.

[11] H. Lenz and K. Wichart, "Variable valve timing-A possibility to control engine load without throttle," SAE Paper No. 880388, 1988.

[12] R. Steinberg, I. Lenz, G. Koehnlein, M. Scheidt, T. Saupe, and W. Buchinger, "A fully continuous variable cam timing concept for intake and exhaust phasing," SAE Paper 980767, 1998.

[13] A. Stefanopoulou, K. Butts, J. Cook, J. Freudenberg, and J. Grizzle, "Consequences of modular controller development for automotivepowertrains: A case study," in Proc. 34th IEEE Conf. Decision Control, 1995, vol. 1, pp. 768-773.

[14] A. G. Stefanopoulou, "Modeling and control of advanced technology engines," Ph.D. dissertation, Dept. Elect. Eng. Comput. Sci., Univ. of Michigan, Ann Arbor, 1996.

[15] B. Powell and J. Cook, "Nonlinear low frequency phenomenological engine modeling and analysis," in Proc. Amer. Control Conf., 1982, vol. 24 , pp. 332-340.

[16] A. Stefanopoulou, J. Freudenberg, and J. Grizzle, "Variable camshaft timing engine control," IEEE Trans. Control Syst. Technol., vol. 8, no. 1, pp. 23-34, Jan. 2000.

[17] M. Cakmakci and A. G. Ulsoy, "Improving component swapping modularity using bi-directional communication in networked control systems," in Proc. Int. Symp. Flexible Automation, Jul. 2006. 